\title{
Is the ring conformation the most critical parameter in lipase-catalysed acylation of cycloalkanols?
}

\author{
Laura M. Levy, Iván Lavandera and Vicente Gotor* \\ Departamento de Química Orgánica e Inorgánica, Facultad de Química, Universidad de Oviedo, 33006-Oviedo, Spain. \\ Fax: +34-985103448; Tel: +34-985103448; E-mail: vgs@fq.uniovi.es.
}

\begin{abstract}
CAL-B catalysed the resolution of several five and six-membered cyclic $\beta$-hydroxy esters efficiently with the exception of the cis-cyclohexanol ( \pm )-4. When employing molecular modelling techniques the conformation turned out to be the most important determinant for their reactivity towards $O$-acetylation. In all cases, the $R$ enantiomers reacted faster than the $S$ enantiomers since the reactive intermediates of the former can adopt more favourable ring conformations and thus experience less steric hindrance in the active site. Furthermore, the minimised structure for the main conformer of $R-\mathbf{4}$ showed that the axial hydrogens in the 3 and 5 positions with respect to the hydroxyl group prevent the enzymatic reaction.
\end{abstract}

\section{Introduction}

The kinetic resolution of racemic mixtures with lipases is an attractive means for the preparation of enantiomerically pure molecules ${ }^{1}$ and is used as a major method for their industrial scale production. ${ }^{2}$

Several 5 and 6-membered cyclic alcohols have been resolved by lipase-catalysed acylation reactions. ${ }^{3,4}$ Amongst them, different 1,2-disubstituted cycloalkanols, which are important chiral building blocks for the asymmetric synthesis of biologically active compounds have been obtained enantiomerically pure using this tool. ${ }^{5,6}$ These resolutions show some common trends, such as, first, the $R$ enantiomer is always preferred by the lipase, secondly, cyclopentanols react more rapidly than cyclohexanols, and thirdly, the cis isomers react more slowly than their trans analogues. During the development of the resolution of several five, six and sevenmembered cyclic cis and trans- $\beta$-hydroxy esters (Figure 1) with lipases A and B from Candida antarctica (CAL-A and CAL-B), we noticed that CAL-B reacted very efficiently with all $R$ enantiomers except in the case of the cis-cyclohexanol. ${ }^{7}$ There are other examples known in the literature in which the cis-2-substituted cyclohexanols do not react with lipases, even when the corresponding cis-2-substituted cyclopentanols do. ${ }^{3 \mathrm{~b}, 8}$ However, in some cases it has been shown that the cis-2-substituted cycloheptanol derivatives react under the same conditions. ${ }^{7,9}$

\section{<insert Figure 1 here>}

Conformational effects could account for this tendency, since in general hydroxyl groups react more slowly in axial than in equatorial position in lipase-catalysed resolutions. ${ }^{3 \mathrm{~b}, 10}$ The same is true for non-biocatalysed transformations. For example, Eliel and Biros showed that the acylation of 2-alkylcyclohexanols occurs faster with the trans isomers (OH equatorial) than with the cis isomers $\left(\mathrm{OH}\right.$ axial) due to steric and polar effects. ${ }^{11}$ Pasto and Rao also recorded a similar trend in 2,5-di-tert-butylcyclohexanol derivatives. ${ }^{12}$

Computer-aided molecular modelling is a potent tool to explain many experimental results in biotransformations. ${ }^{13}$ The force field methods relate the geometry with the potential energy of a molecule using an analytical function. Amongst them, AMBER force field is one of the most commonly used for protein structures. ${ }^{14}$ Conformations can be either randomly or manually generated by rotation of the dihedral angles within the molecule. Crystal structures of the enzyme can be studied with its ligand bound, and both the electrostatic and steric interactions are considered. Thus, modelling of these transition states helps to understand the mechanism, ${ }^{15}$ and the selectivity ${ }^{16}$ of the enzymes.

In this work, we have modelled the intermediates in the CAL-B-catalysed acetylation of five and sixmembered cyclic cis and trans- $\beta$-hydroxy esters (Figure $1, \mathrm{n}=1,2$ ), in order to explain the excellent selectivity seen with the $R$-enantiomers. Together with NMR spectroscopy, the lack of reactivity of the ciscyclohexanol derivative compared to the five-membered analogue was also studied.

\section{Results and Discussion}

\subsection{Resolution of cyclic $\beta$-hydroxy esters by lipase-catalysed acetylation}

As mentioned in the introduction, we have developed the resolution of several five, six, and seven-membered cyclic $\beta$-hydroxy esters via CAL-B-catalysed acetylation with vinyl acetate in tert-butyl methyl ether at $30{ }^{\circ} \mathrm{C}$ 
(Scheme 1). ${ }^{7}$ Under these reaction conditions (Table 1), the acylation of the trans isomers $( \pm)-\mathbf{1}$ and $( \pm)$-2 took place smoothly, yielding both the $S$-substrates and the $R$ products enantiomerically pure. When the same methodology was applied to the cis-configurated $\beta$-hydroxy esters, the $R$ enantiomer of the five-membered derivative $( \pm)-\mathbf{3}$ was rapidly acetylated. However, CAL-B showed very low activity towards cis-cyclohexanol $( \pm)-\mathbf{4}$, obtaining only $5 \%$ conversion after 2 days, despite exhibiting again complete enantiodiscrimination $(E>$ 200).

\section{<insert Scheme 1 here> \\ <insert Table 1 here>}

\subsection{Molecular modelling}

Starting with the X-ray crystal structure of CAL-B (1LBS), ${ }^{18}$ we modelled the analogues of the second tetrahedral intermediate in the acetylation of the cycloalkanols, since this transition state determines the rate and selectivity in a kinetic resolution of racemic alcohols (Figure 2).

In order to simplify the modelling process using molecular mechanics force fields, we chose the phosphonate analogues of these tetrahedral intermediates for minimisation calculations. ${ }^{18}$ Geometry optimisation of the phosphonate containing a methoxy group attached to the phosphorus atom afforded a structure in which all key hydrogen bonds required for catalysis were present (Figure 2). ${ }^{19}$ Next, the whole molecule was added and minimised. Different conformations of these structures were systematically searched for and focused on those most likely to mimic catalytically productive transition states (see under Computational Details). The following two criteria had to be fulfilled: the presence of all key hydrogen bonds within the active site and the lack of steric impediments between the phosphonate and the lipase.

\section{<insert Figure 2 here>}

The active site of CAL-B contains a large hydrophobic pocket (or acyl pocket) above the Asp-His-Ser catalytic triad and a medium-sized pocket (or nucleophile pocket) below it. ${ }^{18}$ The acyl moiety of the intermediate lies in the large subsite (the methyl of the acetate in our case), while the carbocycle of the cycloalkanol binds to the medium-sized pocket. In general, the active site below the catalytic triad restricts the binding of the alcohol substrate to such an extent that only a few conformations were obtained with all the catalytic hydrogen bonds present. ${ }^{19}$ Conformation and ring size proved to be most crucial when determining reactive intermediates.

\subsubsection{Enzymatic acylation of trans-cyclohexanol $( \pm)-2$}

First, we studied the CAL-B-catalysed acetylation of $( \pm)-2$. Cyclohexane derivatives are present in a two chairequilibrium in solution, which lies on the side of the thermodynamically more stable one. Trans-1,2disubstituted cyclohexanes virtually exist only in the conformation that places both substituents in equatorial positions. ${ }^{20}$ Thus, only those structures with both the alcohol and the ester group placed in the less hindered positions, were taken into account during the molecular modelling.

The best structure of the tetrahedral intermediate for the $O$-acetylation of $R-\mathbf{2}$ is shown in Figure 3A. The cyclohexane ring fits perfectly into the medium-sized pocket with all key hydrogen bonds present (Row 1 in Table 2) and with no steric hindrance. Both substituents occupy equatorial positions and the ester moiety lies in the large pocket, with the oxygen of the carbonyl group pointing out towards the solvent.

In the case of the phosphonate analogue of $S$-2, the ring also binds to the medium-sized pocket (Figure 3B). This time however, $\mathrm{H}-2$ and eq-H-3, which are trans to the phosphonate group, are very close to the catalytic histidine despite rotation of both the cyclohexane moiety and the histidine group. As a consequence, three key hydrogen bonds are lost (Row 2 in Table 2).

$$
\begin{aligned}
& \text { <insert Figure 3A here> } \\
& \text { <insert Figure 3B here> } \\
& <\text { insert Table } 2 \text { here> }
\end{aligned}
$$

The different orientations of the substrates in the active site, and the steric hindrance between the cyclohexane and His224 residue, appeared to be a key determinant of the enzyme selectivity. This was also confirmed by the energies obtained for both structures, since the $R$ intermediate was $4.2 \mathrm{kcal}^{\mathrm{mol}}{ }^{-1}$ more stable than the $S$ enantiomer. $^{21}$ 


\subsubsection{Enzymatic acylation of trans-cyclopentanol ( \pm )-1}

Next, we modelled the enzymatic acylation of trans-cyclopentanol $( \pm)-\mathbf{1}$. Conformations of cyclopentanes have been much less studied. Nonetheless, it has been established that there are two symmetrical puckered conformations, the envelope $\left(C_{\mathrm{s}}\right)$ and the half-chair $\left(C_{2}\right)$, with the energy barrier very low between both (Figure 4). The most stable conformer of trans-cyclopentane-1,2-diol is shown in Figure $4 \mathrm{~A}(\mathrm{X}=\mathrm{OH}){ }^{22}$ Normally, five-membered ring derivatives react enzymatically faster than cyclohexanes. ${ }^{3}$ Accelerative factors could be, first, the smaller size of the cycle, which would produce less steric hindrance in the active site, and secondly, the greater ease of cyclopentanes to convert from conformation into another, by this increasing the possibility of finding an acceptable disposition in the active site.

\section{<insert Figure 4 here>}

On the other hand, different conformations of cyclohexanes are more difficult to interconvert. Thus, if the lowest energy chair conformer does not fit into the active site in a successful orientation, it is less probable that the reaction takes place.

When computer modelling was used to minimise the intermediates of the trans-cyclopentane derivatives $( \pm)-\mathbf{1}$, similar results to the six-membered analogues $( \pm)-\mathbf{2}$ were obtained. For $R-\mathbf{1}$, the cycle fits into the medium subsite in a half-chair conformation without any steric impediments (Figure $4 \mathrm{~A}, \mathrm{X}=\mathrm{CO}_{2} \mathrm{Et}$ ), with all key hydrogen bonds present (Row 6 in Table 2). On the contrary, for $S$-1, the ring lies in the medium-sized pocket in a less stable envelope conformer. Furthermore, H-2 and H-3c are too close to His224, destabilising the intermediate by $4.4 \mathrm{kcal}_{\mathrm{mol}}{ }^{-1}$ with respect to the $R$ enantiomer (Row 7 in Table 2). Models are shown in Supplementary Data as Figures S1 and S2.

\subsubsection{Enzymatic acylation of cis-cyclopentanol ( \pm )-3}

The most stable conformation of cis-cyclopentane-1,2-diol is a slightly distorted envelope, shown in Figure 4B $(\mathrm{X}=\mathrm{OH}){ }^{22}$

The phosphonate intermediate shown in Figure 5 was chosen as the best model for the acetylation of $R-\mathbf{3}$. As with the trans isomer, the ring binds perfectly to the medium-sized pocket, with the hydroxyl group in axial position at the fold of the envelope and the ester located in a less hindered equatorial position at the flap of the envelope (Figure $4 \mathrm{~B}, \mathrm{X}=\mathrm{CO}_{2} \mathrm{Et}$ ). This group lies in the large hydrophobic subsite with the oxygen of the carbonyl pointing out towards the solvent. All key hydrogen bonds are present in this structure (Row 8 in Table 2). There are no significant steric interactions in the active site, only $\mathrm{H}-4 \mathrm{c}$ and $\mathrm{H}-5 \mathrm{c}$ being slightly closer to the catalytic histidine (Figure 8A), but not enough to disrupt the interaction between this amino acid and the nucleophile. This may be the cause for the slower reaction with respect to the trans analogue, since the energy of the cis intermediate is $2.4 \mathrm{kcal}_{\mathrm{mol}}{ }^{-1}$ higher.

$$
\begin{aligned}
& \text { <insert Figure } 5 \text { here> } \\
& \text { <insert Figure } 6 \text { here> }
\end{aligned}
$$

When the phosphonate analogue of $S-\mathbf{3}$ was modelled, a structure in which both groups occupied equatorial positions at the fold of the envelope was obtained, with a slightly higher energy (Figure S3 in Supplementary Data). Furthermore, the ester moiety lies too close to the His 224 residue, moving away the cycle towards Thr40. As a result of this, a weaker interaction between this amino acid and the oxyanion takes place (Row 9 in Table 2). This is also confirmed by the energies obtained for both structures, since the $R$ intermediate is 1.7 kcal.mol ${ }^{-1}$ more stable than the $S$ analogue.

\subsubsection{Enzymatic acylation of cis-cyclohexanol ( \pm )-4}

Next, we studied cis-cyclohexanol ( \pm )-4. This substrate proved to be poor for CAL-B, observing almost no product formation even after 2 days. In this case, the ring conformation could be unclear due to the existence of two different chairs which would present one group in axial position, and the other in equatorial position.

${ }^{1}$ H-NMR spectroscopy was used to discern this equilibrium (Figure 6). Several spectra were recorded in three solvents, i.e. $\mathrm{CDCl}_{3}, \mathrm{CD}_{3} \mathrm{CN}$, and acetone- $d_{6}$, the latter two were used as reaction solvents in the enzymatic resolution of ( \pm )-4. ${ }^{7}$ Spectra at $30{ }^{\circ} \mathrm{C}$ and at low temperature were also obtained to study the main conformation. The peak of $\mathrm{H}-1$ ( $\mathrm{H} \alpha$ to the ester) was used in the analysis since it is well resolved as a ddd without any overlapping at 2.4-2.5 ppm. In all the spectra, no signal splitting was observed at low temperature, instead a similar pattern was always seen: one coupling constant of $11-12 \mathrm{~Hz}$ (typical ${ }^{3} J_{\mathrm{HH}}$ ax-ax), and two of 
3-4 Hz (typical ${ }^{3} J_{\mathrm{HH}}$ ax-eq or ${ }^{3} J_{\mathrm{HH}}$ eq-eq). This unequivocally confirms $\mathrm{H}-1$ in axial position with a large ax-ax coupling constant with trans-H-6, and two small ${ }^{3} J_{\mathrm{HH}}$ ax-eq with $\mathrm{H}-2$ and cis-H-6. Thus, in the major conformation of ( \pm )-4 the hydroxyl group lies in axial position. Hence, this more stable conformer of $R-\mathbf{4}$ was investigated first (Figure 7A).

The cyclohexane lies in the medium-sized pocket of the active site and the ester group in the large pocket with the oxygen of the carbonyl pointing out towards the solvent. In this structure, cis-hydrogen atoms in 3,5-diaxial position with respect to the hydroxyl group clash with the catalytic histidine (Figure 8B). These steric hindrances in the active site were enough to disrupt the necessary interaction with the nucleophile, and therefore, the energy of the intermediate is destabilised by $6.4 \mathrm{kcal}^{\mathrm{mol}}{ }^{-1}$ in comparison with the trans isomer $R$-2 (Row 3 in Table 2). These observations can explain the lower reactivity of the cis-R-isomer.

\section{$<$ insert Figure 7A here> \\ $<$ insert Figure 7B here>}

Subsequently, the phosphonate analogue with the hydroxyl group in an equatorial position was built and minimised (Figure 7B). We obtained a structure in which all key hydrogen bonds are present, but the energy of the system is almost $3 \mathrm{kcal}^{\mathrm{mol}}{ }^{-1}$ higher than its conformer 7A (Row 4 in Table 2). This fact could be due to two main factors: first, a higher-energy conformation of the cyclohexane, and secondly, a weaker binding of the substrate within the active site. The ring does not lie in the medium-sized pocket because of steric hindrance between His224 and the ester in axial position, resulting in the loss of stabilising van-der-Waals interactions.

\section{<insert Figure 8 here>}

Lastly, the intermediate of $S \mathbf{- 4}$ was minimised (Figure S4 in Supplementary Data). Although the best structure had a similar energy compared with the one obtained for the $R$ enantiomer, some crucial hydrogen bonds were lost (Row 5 in Table 2), explaining the even lower reactivity of CAL-B towards this enantiomer.

\section{Conclusions}

Although the results of biotransformations are more predictable today than ten years ago, still some trends remain which cannot be explained satisfactorily; e.g. the lack of reactivity of cis-1,2-disubstituted cyclohexanols in lipase-catalysed acylation processes. Using computer-aided molecular modelling, a molecular basis for the acylation of different cyclic $\beta$-hydroxy esters has been proposed in this paper.

The $R$-enantiopreference for these compounds shown by CAL-B is in good agreement with Kazlauskas' rule. ${ }^{23}$ The preferred enantiomers adopt a more favourable ring conformation, in which their binding into the active site is improved. On the contrary, $S$ enantiomers had a worse interaction with the catalytic triad, and crucial hydrogen bonds were lost in some cases.

Furthermore, we have explained the low reactivity of the cis-cyclohexanol derivative. In its major conformation with the hydroxyl group in axial position, the cis-hydrogens in 3,5-diaxial positions impeded the reaction due to steric hindrance. However, the five-membered analogue can react due to the smaller size and the better ring conformation.

The results shown can also explain related experimental facts, such as the lack of reactivity of several trans1,3-disubstituted cyclohexanes. ${ }^{24}$ In this class, the group in 3 position would be preferentially placed in equatorial disposition while the hydroxyl group would be in axial position. The model proposed by us is in accordance with the good reactivity of the cis-2-cyanocyclohexanol with lipases, ${ }^{3 a}$ since small groups in 2 position would favour a ring conformation with an equatorial hydroxyl group. This model can explain the reactivity of cis-1,2- cyclopentanols and of cycloheptanols. The seven-membered analogues could adopt several energetically similar conformers ${ }^{25}$ thus allowing for many different dispositions within the active site of the enzyme, and by this being able to react more easily, despite of their bigger size.

\section{Experimental Section}

\subsection{General}

Compounds ( \pm )-2, $( \pm)-\mathbf{3}$ and $( \pm)-\mathbf{4}$ are commercially available from Aldrich and were used without further purification. Compound $( \pm)-\mathbf{1}$ was synthesised using a procedure similar to that reported by Nakata and 
Oishi. ${ }^{7,26}{ }^{1} \mathrm{H}-\mathrm{NMR}$ spectra were obtained with a Bruker AMX 400 (400.13 MHz) spectrometer, using $\mathrm{CDCl}_{3}$, $\mathrm{CD}_{3} \mathrm{CN}$ and acetone- $d_{6}$ as solvents.

\subsection{Computational Details}

The program Insight II, version 2000.1, was used for viewing the structures. The geometric optimisations were performed using Discover, version 2.9.7 (Accelrys, San Diego CA, USA), using the AMBER force field. ${ }^{14}$ The distance dependent dielectric constant was set to 4.0 and the 1-4 van-der-Waals interactions were scaled to $50 \%$. The crystal structure of CAL-B (1LBS) ${ }^{18}$ was obtained from the Protein Data Bank (www.rcsb.org/pdb/).

Hydrogen atoms were added to the structure, tested for partial charge balance and corrected for atom types within the AMBER force field. The $\mathrm{pH}$ of the catalytic histidine was adjusted to $\mathrm{pH} 4$ and the structure was then relaxed. Geometry optimisations were carried out in two steps. First, the steepest descent algorithm got the molecule to a local minimum with a RMS deviation of about $0.02 \AA . \mathrm{mol}^{-1}$. Secondly, conjugate gradient algorithm gave a more precise minimisation near the local minimum, obtaining a RMS value of $0.005 \AA$ A.mol ${ }^{-1}$.

Next, a phosphonate core of the tetrahedral intermediate mimicking methylation of methanol was assembled in the crystal structure. Partial charges on the intermediate atoms were set to those of the tetrahedral intermediate (carbon, not phosphorus), obtaining the values by semi-empirical calculation performed using Chem3D using the AM1 parameters.

For the optimisation of this phosphonate core a systematic approach was applied. Initially, the intermediate was allowed to adjust to the enzyme active site by keeping the entire enzyme fixed during the geometry optimisation. The side chains of the lipase were then released and allowed to adjust. Finally, the entire complex was allowed to adjust. This approach avoided drastic changes in the lipase structure caused by nonoptimal conformations of the intermediate until an RMS maximum value of $0.005 \AA \mathrm{mol}^{-1}$ was obtained. A hydrogen bond calculation was performed after each minimisation in order to assure the presence of the critical hydrogen bonds around the catalytic site. ${ }^{19}$

$$
\text { <insert Figure } 9 \text { here> }
$$

The rest of the molecule was added and different conformations were produced by manual adjustment of dihedral angles specified in Figure 9. Geometry optimisations were again performed using the same approach described above. Due to the flexible structure of these intermediates, small adjustments of the dihedral angle $a$ caused large changes in the orientation of the ester moiety. Adjustments of approximately $10-20^{\circ}$ were performed for both dihedral angles adjacent to that position. Structures causing obvious steric hindrance were ignored.

Acknowledgements. Financial support of this work by the Spanish Ministerio de Ciencia y Tecnología (Project No. PPQ-2001-2683) and by Principado de Asturias (Project No. GE-EXP01-03) is gratefully acknowledged. I.L. thanks MCYT for a pre-doctoral fellowship.

Supplementary Data Available. Complete ${ }^{1} \mathrm{H}-\mathrm{NMR}$ spectral data are shown. Furthermore, some extensive modelling figures are included. This material is available free of charge via the Internet at http://www.rsc.org/esi.

\section{References}

1. a) C. J. Sih and S.-H. Wu, Top. Stereochem., 1989, 19, 63; b) U. T. Bornscheuer and R. J. Kazlauskas, Hydrolases in Organic Synthesis: Regio- and Stereoselective Biotransformations; Wiley-VCH: Weinheim, 1999.

2. A. Liese, K. Seelbach and C. Wandrey, Industrial Biotransformations; Wiley-VCH: Weinheim, 2000.

3. a) E. Forró, K. Lundell, F. Fülöp and L. T. Kanerva, Tetrahedron: Asymmetry, 1997, 8, 3095; b) A. Maestro, C. Astorga and V. Gotor, Tetrahedron: Asymmetry, 1997, 8, 3153; c) A. Luna, C. Astorga, F. Fülöp and V. Gotor, Tetrahedron: Asymmetry, 1998, 9, 4483; d) E. Forrö, Z. Szakonyi and F. Fülöp, Tetrahedron: Asymmetry, 1999, 10, 4619; e) L. M. Levy and V. Gotor, J. Org. Chem., 2004, 69, 2601. 
4. J. González-Sabín, V. Gotor and F. Rebolledo, Tetrahedron: Asymmetry, 2004, 15, 1335.

5. J. K. Whitesell, Chem. Rev., 1992, 92, 953.

6. a) D. H. Apella, L. A. Christianson, D. A. Klein, D. R. Powell, X. Huang, J. Barchi, Jr. and S. H. Gellman, Nature (London), 1997, 387, 381; b) A. Pecunioso, M. Maffeis, C. Marxhioro, L. Rossi and B. Tamburini, Tetrahedron: Asymmetry, 1997, 8, 775; c) E. Racanska and F. Gregan, Pharmazie, 1999, 54, 68.

7. L. M. Levy, J. R. Dehli and V. Gotor, Tetrahedron: Asymmetry, 2003, 14, 2053.

8. a) E. Forró, L. T. Kanerva and F. Fülöp, Tetrahedron: Asymmetry, 1998, 9, 513-520; b) C. Brunet, M. Zarevucka, Z. Wimmer and M.-D. Legoy, Enzyme Microb. Technol., 2002, 31, 609.

9. E. Forró and F. Fülöp, Tetrahedron: Asymmetry, 1999, 10, 1985.

10. R. Tanikaga, Y. Matsumoto, M. Sakaguchi, Y. Koyama and K. Ono, Tetrahedron Lett., 2003, 44, 6781.

11. E. L. Eliel and F. J. Biros, J. Am. Chem. Soc., 1966, 88, 3334.

12. D. J. Pasto and D. R. Rao, J. Am. Chem. Soc., 1970, 92, 5151.

13. a) R. J. Kazlauskas, Curr. Opin. Chem. Biol., 2000, 4, 81; b) R. Kazlauskas, Science, 2001, $293,2277$.

14. a) S. J. Weiner, P. A. Kollman, D. A. Case, U. C. Singh, C. Ghio, G. Alagona, S. Profeta and P. Weiner, J. Am. Chem. Soc., 1984, 106, 765; b) W. D. Cornell, P. Cieplak, C. I. Bayly and P. A. Kollmann, J. Am. Chem. Soc., 1993, 115, 9620.

15. S. Park, E. Forró, H. Grewal, F. Fülöp and R. J. Kazlauskas, Adv. Synth. Catal., 2003, 345, 986.

16. a) F. Hæffner and T. Norin, Chem. Pharm. Bull., 1999, 47, 591; b) K. Hult and P. Berglund, Curr. Opin. Biotechnol., 2003, 14, 395.

17. C. S. Chen, Y. Fujimoto, G. Girdaukas and C. J. Sih, J. Am. Chem. Soc., 1982, 104, 7294.

18. J. Uppenberg, N. Öhrner, M. Norin, K. Hult, G. J. Kleywegt, S. Patkar, V. Waagen, T. Anthonsen and T. A. Jones, Biochemistry, 1995, 34, 16838.

19. To identify hydrogen bonds, a donor atom to acceptor atom distance of less than $3.20 \AA$ and a donor atom hydrogen - acceptor atom angle of $120^{\circ}$ or greater are required.

20. Several ${ }^{1}$ H-NMR spectral data of $( \pm$ )-2 were recorded in different solvents, and signals of $\mathrm{H}-1$ ( $\alpha$ to the ester) and $\mathrm{H}-2$ ( $\alpha$ to the hydroxyl group), showed two ${ }^{3} J_{\mathrm{HH}}$ ax-ax (between $10-14 \mathrm{~Hz}$ ) and one ${ }^{3} J_{\mathrm{HH}}$ between 3$5 \mathrm{~Hz}$, typically ax-eq. This indicates that both hydrogens must be in axial positions.

21. The relation between the difference in the Gibbs energy of the transition states of the enantiomers and the enantiomeric ratio can be shown with the transition state theory to be $\Delta_{R-S} \Delta \mathrm{G}^{\ddagger}=\Delta \mathrm{G}_{R}{ }^{\ddagger}-\Delta \mathrm{G}_{S}{ }^{\ddagger}=-\mathrm{RT} \ln E$.

22. R. J. Abraham, R. Koniotou and F. Sancassan, J. Chem. Soc., Perkin. Trans. 2, 2002, 2025.

23. R. J. Kazlauskas, A. N. E. Weissfloch, A. T. Rappaport and L. A. Cuccia, J. Org. Chem., 1991, 56, 2656.

24. a) R. Tanikaga and A. Morita, Tetrahedron Lett., 1998, 39, 635; b) L. M. Levy, G. de Gonzalo and V. Gotor, Tetrahedron: Asymmetry, 2004, 15, 2051-2056.

25. a) D. F. Bocian and H. L. Strauss, J. Am. Chem. Soc., 1977, 99, 2876; b) K. B. Wiberg, J. Org. Chem., 2003, 68, 9322.

26. T. Nakata and T. Oishi, Tetrahedron Lett., 1980, 21, 1641. 
Table 1 Resolution of cyclic $\beta$-hydroxy esters with CAL-B. ${ }^{a}$

\begin{tabular}{ccccc}
\hline $\mathrm{n}^{b}$ & Substrate & $t(\mathrm{~h})$ & $c^{c}$ & $E^{c}$ \\
\hline 1 (trans) & $( \pm)-\mathbf{1}$ & 1.3 & 50 & $>200$ \\
$2($ trans $)$ & $( \pm)-\mathbf{2}$ & 1.7 & 50 & $>200$ \\
$1($ cis $)$ & $( \pm)-\mathbf{3}$ & 2.7 & 50 & $>200$ \\
$2($ cis $)$ & $( \pm)-\mathbf{4}$ & 48 & 5 & $>200$
\end{tabular}

${ }^{a}$ See Ref. 7. ${ }^{b}$ See Scheme $1 .{ }^{c}$ Calculated from the e.e. of the substrate and the product. See Ref. 17.

Table 2 Key hydrogen bonds and energy in CAL-B-catalysed acetylation of cyclic trans- and cis- $\beta$-hidroxy esters of five and six members. ${ }^{a}$

\begin{tabular}{|c|c|c|c|c|c|c|c|c|c|}
\hline \multirow[t]{2}{*}{ Row } & \multirow[t]{2}{*}{ Compound } & \multirow[t]{2}{*}{ Figure $^{b}$} & \multicolumn{6}{|c|}{ H-bond distance, $\AA$ (angle) ${ }^{c}$} & \multirow[t]{2}{*}{$\mathrm{E}\left(\mathrm{kcal} \cdot \mathrm{mol}^{-1}\right)$} \\
\hline & & & $\mathrm{a}$ & $\mathrm{b}$ & $\mathrm{c}$ & $\mathrm{d}$ & $\mathrm{e}$ & $\mathrm{f}$ & \\
\hline 1 & $(2 R)-2$ & $3 \mathrm{~A}$ & $3.02\left(134^{\circ}\right)$ & $3.14\left(131^{\circ}\right)$ & $2.91\left(147^{\circ}\right)$ & $3.03\left(160^{\circ}\right)$ & $2.75\left(168^{\circ}\right)$ & $2.82\left(170^{\circ}\right)$ & 0 \\
\hline 2 & $(2 S)-2$ & $3 B$ & $3.25\left(124^{\circ}\right)$ & $3.31\left(134^{\circ}\right)$ & $2.91\left(133^{\circ}\right)$ & $3.39\left(162^{\circ}\right)$ & $2.76\left(168^{\circ}\right)$ & $2.85\left(168^{\circ}\right)$ & $+4.2^{d}$ \\
\hline 3 & $(2 R)-\mathbf{4}(\mathrm{OH}$ ax $)$ & $7 \mathrm{~A}$ & $3.06\left(132^{\circ}\right)$ & $3.01\left(146^{\circ}\right)$ & $3.40\left(139^{\circ}\right)$ & $2.97\left(161^{\circ}\right)$ & $2.78\left(172^{\circ}\right)$ & $2.83\left(166^{\circ}\right)$ & $+6.4^{d}$ \\
\hline 4 & $(2 R)-4(\mathrm{OH}$ eq $)$ & $7 \mathrm{~B}$ & $3.07\left(150^{\circ}\right)$ & $2.96\left(128^{\circ}\right)$ & $2.95\left(139^{\circ}\right)$ & $3.16\left(155^{\circ}\right)$ & $2.80\left(165^{\circ}\right)$ & $2.78\left(166^{\circ}\right)$ & $+9.3^{d}$ \\
\hline 5 & $(2 S)-4$ & S4 & $3.48\left(126^{\circ}\right)$ & $2.95\left(127^{\circ}\right)$ & $3.27\left(113^{\circ}\right)$ & $3.18\left(160^{\circ}\right)$ & $2.72\left(167^{\circ}\right)$ & $3.03\left(165^{\circ}\right)$ & $+5.9^{d}$ \\
\hline 6 & $(2 R)-\mathbf{1}$ & S1 & $3.03\left(135^{\circ}\right)$ & $3.16\left(129^{\circ}\right)$ & $2.83\left(144^{\circ}\right)$ & $3.01\left(157^{\circ}\right)$ & $2.77\left(172^{\circ}\right)$ & $2.80\left(172^{\circ}\right)$ & 0 \\
\hline 7 & $(2 S)-\mathbf{1}$ & $\mathrm{S} 2$ & $3.23\left(127^{\circ}\right)$ & $3.32\left(139^{\circ}\right)$ & $2.85\left(139^{\circ}\right)$ & $3.55\left(165^{\circ}\right)$ & $2.76\left(161^{\circ}\right)$ & $2.79\left(159^{\circ}\right)$ & $+4.4^{e}$ \\
\hline 8 & $(2 R)-\mathbf{3}$ & 5 & $3.01\left(134^{\circ}\right)$ & $2.91\left(131^{\circ}\right)$ & $3.06\left(149^{\circ}\right)$ & $2.85\left(154^{\circ}\right)$ & $2.77\left(174^{\circ}\right)$ & $2.85\left(175^{\circ}\right)$ & $+2.4^{e}$ \\
\hline 9 & $(2 S)-\mathbf{3}$ & S3 & $3.00\left(133^{\circ}\right)$ & $3.15\left(132^{\circ}\right)$ & $2.93\left(155^{\circ}\right)$ & $3.09\left(161^{\circ}\right)$ & $2.78\left(168^{\circ}\right)$ & $3.16\left(128^{\circ}\right)$ & $+4.1^{e}$ \\
\hline
\end{tabular}

${ }^{a}$ Lost hydrogen bonds appear in red. ${ }^{b}$ See text. ${ }^{c}$ Figure 2 defines the hydrogen bonds. ${ }^{d}$ Compared with the energy of Figure 3 A. ${ }^{e}$ Compared with the energy of Figure S1. 